EPJ Web of Conferences 41, 09006 (2013)

DOI: $10.1051 /$ epjconf/20134109006

C) Owned by the authors, published by EDP Sciences, 2013

\title{
Near-field terahertz imaging of a discontinuity in split ring resonator array
}

\author{
F. Blanchard ${ }^{1,2}$, K. Ooi ${ }^{1}$, T. Tanaka ${ }^{1,2}$, A. Doi ${ }^{3}$ and K. Tanaka ${ }^{1,2,4}$ \\ ${ }^{1}$ Institute for Integrated Cell-Material Sciences (WPI-iCeMS), Kyoto University, Kyoto, Japan \\ ${ }^{2}$ CREST, Japan Science and Technology Agency, Kawaguchi, Saitama, Japan \\ ${ }^{3}$ Olympus Corporation 2-3 Kuboyama-cho, Hachioji-shi, Tokyo, Japan \\ ${ }^{4}$ Department of Physics Graduate School of Science, Kyoto University, Kyoto, Japan
}

\begin{abstract}
We investigated the spatiotemporal evolution of single cycle terahertz pulses transmitted through a split ring resonator array including a void. Using a large-field-ofview terahertz microscope, we revealed the confinement and enhancement of the defect mode.
\end{abstract}

\section{Introduction}

Metamaterials have attracted a lot of attention due to their unique ability to be engineered to have the desired electromagnetic properties at optical wavelengths [1]. These artificial materials have exotic properties such as negative magnetic permeability, perfect lensing, and cloaking [1]. The most common metamaterial is an array of split ring resonators (SRR), where the unit cell is small compared with the wavelength and consists of metallic rings each with a small gap [2]. In SRRs organized in a periodic fashion, the coupling between elements leads the material to behave like a photonic crystal with electromagnetic modes propagating in the array, i.g., topic related to magnetoinductive wave [3].

A lot of study has gone into making photonic crystals with a high-Q resonant mode inside the band-gap [4]. The defect mode, or cavity resonant mode, should also be present in an SRR array that has properties like those of photonic crystal. However, as yet, there has been no investigation of how the electromagnetic field distribution evolves in the presence of defects in a SRR array.

In this study, we investigated the effect of defects inside an SRR array by visualizing the electromagnetic field distributions at terahertz $(\mathrm{THz})$ frequencies. The recent technique of near-field $\mathrm{THz}$ microscopy was used for this purpose. In particular, we recorded $\mathrm{THz}$ images of the electric field distribution inside the cavity and for several SRR neighbors. The field was enhanced at the resonance frequency inside the space left by one missing element. To our knowledge, this is the first electric field visualization of a defect mode in the $\mathrm{THz}$ region (including the defect mode in photonic crystals).

\section{Experimental}

We used a THz microscope, as previously reported [5]. A large probe beam at $800 \mathrm{~nm}$ illuminated a $\mathrm{LiNbO}_{3}(\mathrm{LN})$ crystal used for the purpose of two-dimensional electro-optic (EO) imaging in the reflection scheme. When dealing with $\mathrm{THz}$ images, a very thin $\mathrm{EO}$ crystal is needed in order to

This is an Open Access article distributed under the terms of the Creative Commons Attribution License 2.0, which permits unrestricted use, distribution, and reproduction in any medium, provided the original work is properly cited. 
preserve the high spatial resolution of the optical elements. This constraint enables the $\mathrm{THz}$ field to be collected from a sample before diffraction [5]. The losses in sensitivity caused by a thin EO crystal were compensated by a tightly focused pulse ranging from 0.1 to $2.5 \mathrm{THz}$ and with a peak field of $600 \mathrm{kV} / \mathrm{cm}$ [6]. In addition, using a two-dimensional EO near-field imaging technique prevents any perturbation from an external metallic structure, e.g., the tip used for the raster scanning near-field technique. Thus, the $\mathrm{THz}$ field localized inside the cavity along with amplitude and phase changes induced in its neighbors can be accurately and simultaneously measured.

Figures 1-(a) and (b) show a visible image and an illustration of a metallic mask fabricated on top of a $1 \mu \mathrm{m}$-thick $\mathrm{x}$-cut $\mathrm{LN}$ crystal sensor to evaluate the spatial resolution at $\mathrm{THz}$ frequencies. By Fourier transforming the measured temporal evolution of the electric field passing through the sample, we obtained the spatial resolution as a function of frequency. The components at $1 \mathrm{THz}$ and $0.24 \mathrm{THz}$ are presented in Fig. 1-(c) and (d), respectively. As shown in Fig. 1-(d), the line and space pattern is completely resolved, which confirmed that the spatial resolution was better than $\lambda / 125$.

(a)

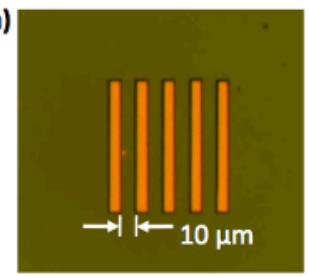

(c)

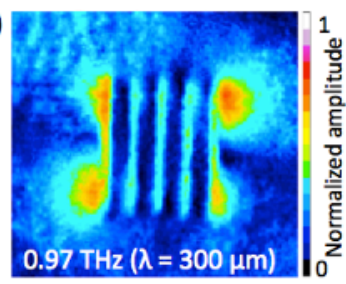

(b)
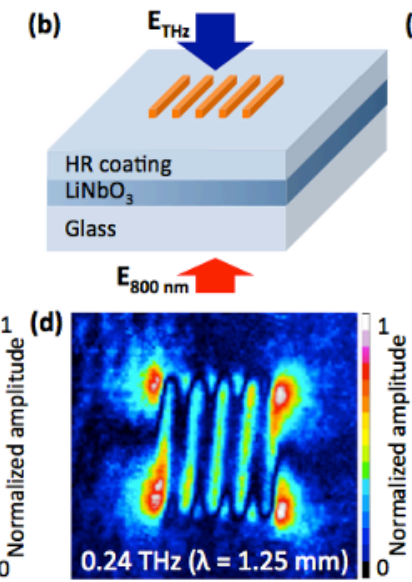

(e)

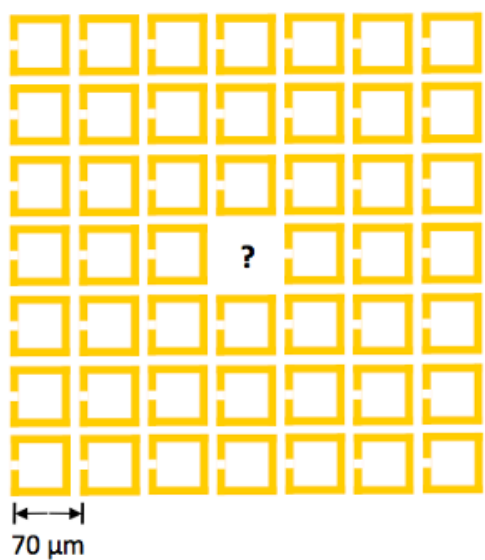

Fig. 1. (a) Visible image of a line and space metallic pattern; (b) illustration of the measurement technique.; (c) and (d) Fourier components at $1 \mathrm{THz}$ and $0.24 \mathrm{THz}$ of the measured temporal evolution of the electric field passing through the sample; (e) schematized array of SRRs including a void.

To explore the defect mode at $\mathrm{THz}$ frequencies, we fabricated an array of $23 \times 23$ SRRs with 70 $\mu \mathrm{m}$ lattice and removed one element in its center (Fig.1-(e)). The THz excitation polarization and the probe beam $\left(\lambda_{800 \mathrm{~nm}}\right)$ polarization were set parallel to each other and parallel to the gap. The sample was directly patterned on the high-reflecting-coating side of a $3 \mu \mathrm{m}$-thick $\mathrm{x}$-cut LN crystal by using standard photolithography and lift-off techniques on a $100 \mathrm{~nm}$-thick deposition of gold. A $50 \mu \mathrm{m}$ long, $9.5 \mu \mathrm{m}$ wide square shape and a $5 \mu \mathrm{m}$ gap composed each SRR element inside the array.

\section{Results}

Figure 2 shows two aspects of the defect mode in the SRR array that relates the temporal near-field evolution of the $\mathrm{THz}$ pulse passing through the samples (with and without a void) and the visualization of the enhancement found inside the void. The left-hand and right-hand sides of Fig. 2-(a) show the time-domain near-field THz images of a SRR array with and without a void, respectively. Both movies (SRR arrays with and without the defect) are 300 frames long, with a 27fs time step. 
(a)

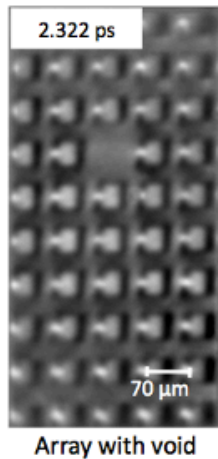

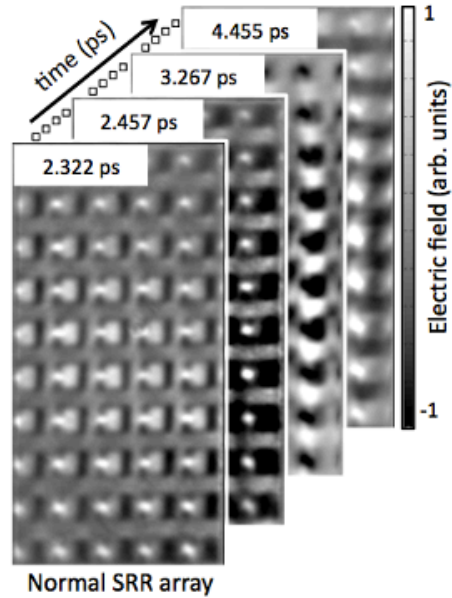

(b)
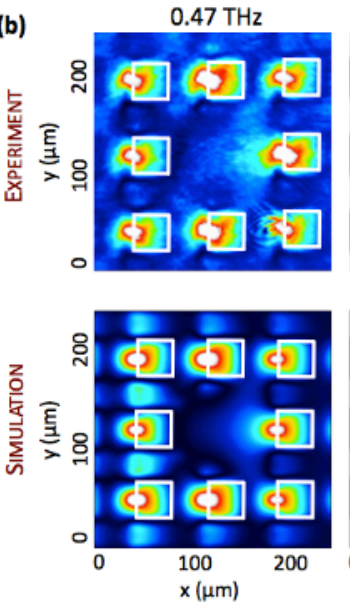

$1.35 \mathrm{THz}$
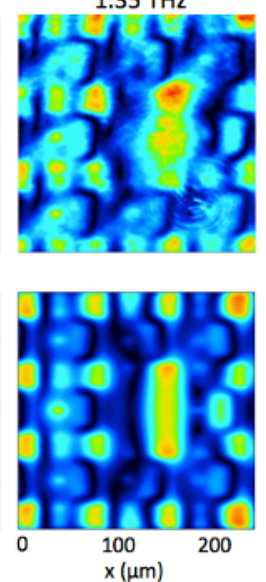

Fig. 2. (a) Near-field $\mathrm{THz}$ electric field distributions from SRR arrays with and without a missing element; (b) experimental and simulated Fourier transformed near-field maps at $0.47 \mathrm{THz}$ and $1.35 \mathrm{THz}$.

Figure 2-(b) shows the magnitude of the Fourier transformed near-field maps at $0.47 \mathrm{THz}$ and $1.35 \mathrm{THz}$, which correspond to the lowest resonant mode of the SRR array together with one cavity resonance found inside the void. To validate our measurements, we used finite domain time difference (FDTD) software that reproduced the experimental conditions. The lower part of Fig. 2(a) represents the corresponding simulated frequencies, which perfectly match our measurements. The narrow band resonant frequencies found inside the cavity exhibited an enhancement factor of 2 and are not confined symmetrically inside the void; i.e., the strongest amplitude is not at its center. This may be due to the asymmetric shape of the unit cell itself.

\section{Discussions}

We conducted a near-field $\mathrm{THz}$ study on metamaterials where one element was removed from an array in order to elucidate the electromagnetic field distribution inside the discontinuity. Our system successfully identified the resonant frequency of the SRR array and the resonant modes created inside the void space. This work provides the first insight on defect modes in two-dimensional metamaterial arrays. A deeper analysis of the role and merits of defect modes in SSR arrays will be part of a further publication.

\section{References}

1. D. R. Smith, J. B. Pendry, and M. C. K. Wiltshire, Science 305, 788-792 (2004)

2. J. B. Pendry, A. Holden, D. Robbins, and W. Stewart, IEEE Trans. Microwave Theor. Tech. 47 (11), 2075 (1999)

3. E. Shamonia, V. A. Kalinin, K. H. Ringhofer, and L. Solymar, J. Appl. Phys. 92, 6252-6261 (2002)

4. Y. Akahane, T. Asano, B.-S. Song, and S. Noda, Nature 425, 944-947 (2003)

5. F. Blanchard, A. Doi, T. Tanaka, H. Hirori, H. Tanaka, Y. Kadoya, and K. Tanaka, Opt. Express 19, 8277-8284 (2011)

6. H. Hirori, A. Doi, F. Blanchard, and K. Tanaka, Appl. Phys. Lett. 98, 091106 (2011) 